\title{
Fault tolerant CMOS logic using ternary gates.
}

\author{
Yngvar Berg, Rene Jensen, Johannes Lomsdalen, Henning Gundersen \\ Snorre Aunet \\ Microelectronic Systems \\ Department of Informatics \\ University of Oslo \\ Oslo, Norway \\ Email: yngvarb@ifi.uio.no
}

\begin{abstract}
In this paper we present fault tolerant CMOS logic using redundancy and ternary signals. The ternary gates are implemented using recharge logic which can be exploited in binary and multiple-valued logic (MVL). Signals are processed through capacitors in such a way that the logic operation of a gate is independent of the DC voltage applied on the inputs. By combining signals through capacitors stuck on/stuck off and stuck at faults are not destructive when redundancy is applied. Simulated data for $130 \mathrm{~nm}$ and $0.35 \mu \mathrm{m}$ CMOS processes are given.
\end{abstract}

\section{Introduction}

The technology trend introduces a wide variety of problems related to reliability of circuits. The ever increased integration of devices on a single die raises the probability of erroneous components in the die. The increasing probability of faults in a circuit increase the demand of fault tolerant logic. The most reasonable way to overcome the reliability problems is to build fault tolerant circuits. Floating-gate CMOS[1] can be used to overcome some of the problems due to stuck-at failures.

In this paper we present a ternary logic gate which is fault tolerant. I section II we discuss briefly some common faults in CMOS circuits, and in section III we introduce a semi-floating-gate[2] (SFG) majority-3 (MAJ3) ternary inverter which reduces the effect of faults compared to standard CMOS gates. In section IV we present a fault tolerant redundant ternary SFG gate.

\section{Faults and redundancy}

In this section we will discuss briefly common CMOS failures[3]. The failures considered are

- Stuck-at faults.

- Stuck at $V_{D D}$ (SA1). A node is assumed to be stucked at $V_{D D}$ independently of signal values or other failures. We assume that the node will not be affected by currents pulling the the node towards gnd. The failures can be caused by defect pMOS transistor(s) pulling a node to $V_{D D}$ with a current larger than the $\mathrm{ON}$ current provided by operative transistors.

- Stuck at gnd (SA0). A node is assumed to be stucked at $g n d$ independently of signal values or other failures. We assume that the node will not be affected by currents pulling the the node towards $V_{D D}$.

\section{- Stuck-open faults.}

- MOSFET stuck-open (SOFF). A transistor that can not be turned on, is assumed to deliver a minimum current given by the OFF state of a transistor.

- Interconnect stuck-open (IOFF). Floating nodes. Normally modelled by unconnected transistor terminals. An unconnected gate terminal may be equivalent to a SOFF or even a SON failure.

- Stuck-closed faults.

- MOSFET stuck-closed (SON). A transistor that can not be turned off, is assumed to deliver a maximum current given by the $\mathrm{ON}$ state of a transistor. 


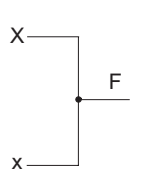

(a)

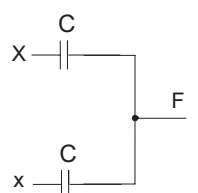

(b)
Figure 1. Redundancy by (a) connecting two redundant signals $X$ and $x$ directly (wired-or), and (b) connecting two redundant signals through capacitive connections.

\section{- Resitive shorts.}

- MOSFET shorts. Equivalent to a SON failure.

- Shorts to $V_{D D}$. Equivalent to SA1 failure.

- Shorts to gnd. Equivalent to SA0 failure.

- Shorts between nodes (bridge) (RBR). Resistive bridge between nodes, gate outputs or feedback.

In addition we have to consider faults associated with floating capacitors:

- Floating capacitor stuck-open (COFF). An input capacitor with zero capacitance. Equivalent to a input stuck at (SA) fault.

- Floating capacitor stuck-closed (CON). Resistive connection between an input signal and a floating gate.

Redundancy has been used as a general method to overcome failures in CMOS circuits. The most common redundancy realization is a triple modular redundancy[4].

\subsection{Redundancy}

Transistor open and closed failures can be compensated for by using redundancy and simply wiring together redundant gates[4]. Stuck-at failures, however, are not necessarily compensated for by this strategy due to limited driving capability of the transistors.

As shown in Figure 1 (a) redundancy is implemented by connecting two (or more) signals. In this case the result is determined by the driving capabilities of the gates proving signals $X$ and $x$. If one of the signals is stuck-at 0 or 1 we can assume that the stuck-at failure will dominate and the redundant (and normal operative) gate will not provide a correct logic level for $F$. In the case of a SON failures two redundant gates may be pulling $F$ in opposite directions with the same current levels and thus leaving a unknown or intermediate logic level at $F$. If three or more redundant gates are used a SON failure will not be visible at $F$ providing that the input signals to the gates are correct. Bridge faults may determine the signal value of $F$ dependent on the bridge resistance, driving capabilities of the gates involved and/or faults associated with the respective gates. Bridge failures (RBR) share some similarities with cross talk noise and can reduce robustness and noise margins in a system. The node $F$ is not likely to experience a bit error due to bridge failures or cross talk directly. However due to the a severe degradation of the time constant as a consequence of this failures a signal $F$ may not respond fast enough compared to the operating frequency of the system, and $F$ may therefor be interpreted incorrectly by the preceding gate. By connecting two or more gates, errors due to SOFF and IOFF faults can be avoided.

In Figure 1 (b) redundancy is implemented by connecting two (or more) signals through capacitive connections. In the case of stuck-at failures associated with $X$ or $x$ the driving capabilities of the two redundant gates providing these signals will not dominate the node $F$ significantly. If $X$ is stuck at 0 or 1 then a voltage change at $x$ will be transfered to $F$ through the floating capacitor. The change in $F$ will be less than if both $X$ and $x$ changes in the same direction. The only severe problem may arise if $X$ and $x$ changes in opposite directions. In addition we may have to consider a possibility of a stuck at fault at $F$ itself. In the case of SON and SOFF failures the same arguments can be applied. There will only be a significant problem if the redundant signals switches in opposite directions. In the case of bridge (RBR) faults the combination of signals through capacitive connections will reduce the probability of an error.

\section{Ternary recharge logic}

Any floating-gate (FG) circuit need to be initialized, either once or frequently. By recharging a SFG frequently we avoid problems with any leakage currents and random or undesired disturbance of the floating-gate charges. When reseting or recharging a gate the inputs are recharged simultaneously and not set to a reference voltage, normally $V_{s s}$ or $V_{d d}$. While recharging, the semi-floating-gate of a logic gate is forced to $V_{d d} / 2$. The clocked-Neuron-MOS logic was proposed for binary logic gates and for threshold gates $[5,6]$. The recharge scheme is similar to biasing of single-ended auto-zeroing comparators which have been used in high-speed flash AD converters. The main purpose of the recharge scheme is to initialize or recharge the semifloating-gates to an equilibrium state which can be utilized to yield fast binary and multiple-valued signal processing [2]. In addition we may reduce the effect of mismatches, especially transistor mismatches, and power supply noise. The recharge scheme provides a simple, fast and accurate recharge to the equilibrium state for all gates regardless of logical depth. The logic gate output are recharged to a spe- 


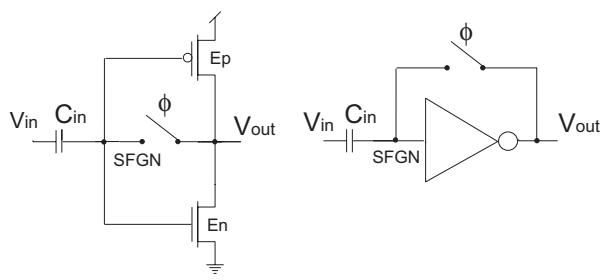

Figure 2. $S F G$ inverter.

cific value, namely $V_{d d} / 2$ and the SFG are recharged to the initial equilibrium state, namely $V_{d d} / 2$.

The precharge/recharge voltage is $V_{D D} / 2$ witch is neither logic 0 or 1 . The recharge level can be defined as $1 / 2$, or alternatively 0 if voltage level 0 is defined as -1 and $V_{D D}$ is defined as 1 . This is called balanced ternary notation. The input signal to a SFG gate is not processed through its level, the change in voltage level from the recharge level determines the change in the output of the gate. Normally the inputs signals will be +1 or -1 . If, however, the input is fixed to any voltage level, both during recharge and evaluate, there will be no change and the input will not influence on the logical operation of the gate.

\subsection{Faults}

We can analyze the SFG inverter, shown in Figure 2, in terms of response to an internal fault, assuming that the input signal is correct. The transistor faults can be located at the output $E_{p}$ or $E_{n}$ or in the switch providing the feedback from the output to the semi floating gate node (SFGN).

\subsubsection{Fault in the switch (TG)}

A SOFF fault in the switch will not influence significantly on the operation of the circuit if the switch is a transmission gate (TG). The effect of a SOFF in one of the feedback transistors will be visible both as a minor deviation from the defined initial state (logic 0 ) and an increased recharge delay.

A SON fault in one of the recharge transistors will effectively pull the SFGN and the output towards the initial state at a rate determined by the driving capabilities or effective resistance of the transistor with the SON fault. We can assume that the gate will respond to an input change with a voltage change in the right direction, the response will, however, be less than expected and temporary. A SON fault in the transmission gate will not result in a output change in the wrong direction. The most severe error is that the gate will not respond to an input signal and thus the output will be stucked at $V_{D D} / 2$ (SA). Note that any static signal, independently of the static voltage will be received and interpreted equivalently. A feedback bridge is equivalent to a TG SON fault.

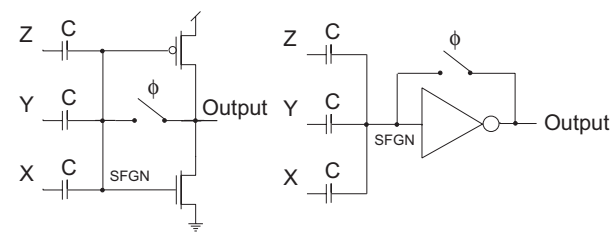

Figure 3. Ternary majority-3 gate.

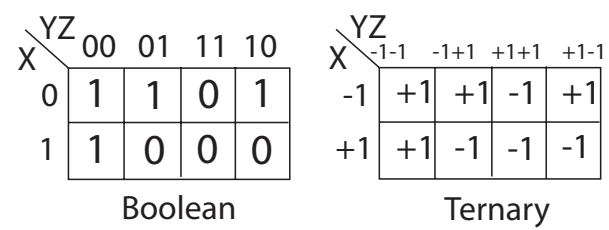

Figure 4. Boolean and ternary majority-3 gate function. A ternary 0 implies an error due to a fault.

\subsubsection{Faults in the output transistors}

If one of the output transistors is SOFF the output will be precharged close to the supply rail of the normally operating transistor. The gate will only respond marginally to an input signal. The voltage change will be in the correct direction. In the case where one of the output transistor is SON a similar situation will occur. The precharge value will be close to the supply feeding the SON transistor and the gate will respond to an input signal with a attenuated voltage change in the correct direction.

\subsubsection{Interconnect stuck-open}

The gate will respond to this failure similar to SOFF fault in one of the output transistors. The IOFF fault will effectively generate a static output voltage.

\subsection{SFG ternary majority-3 gate}

A ternary majority-3 SFG gate, with two transistors, is shown in Figure 3. The increased complexity in logic operation is not dependent on an increased number of transistors and thus the probability of transistor faults will not increase with the complexity of the gate. We have to consider possible faults associated with the capacitors. There are two faults to be considered, namely open (COFF) and closed (CON). For a single input SFG inverter a $\mathrm{CON}$ fault will actually be beneficial because the input signal will not be attenuated. A COFF fault may further lead to a leakage problem and a voltage change possibly in the wrong direction. For a multiple input SFG gate a CON fault may result in a logic error. If $X=Y=+1$ and $Z=-1$ the correct operation of the gate is -1 , if however, the ca- 
pacitor connected to $Z$ is CON the output will most probably be +1 . This error depends on timing details. Normally the gate providing the input signal $X$ will see a capacitance equal to $C$ in addition to parasitic capacitance $\left(C_{\text {par }}\right)$ in the gate itself. If the capacitor connecting $X$ to the SFGN is CON the load capacitance seen by the input $X$ will be increased from $C+C_{\text {par }}$ to $3 C+C_{\text {par }}+2 C_{g}+2 C_{\text {diff }}$ where $2 C_{g}+2 C_{d i f f} \approx C_{\text {par }_{X}}$ and $C>C_{\text {par }}$. Hence the load capacitance has been tripled and therefore the timing response is changed. If the frequency is high the extra load on $X$ may reduce the effect of a CON fault. If the CON resistance is high compared to a transistor resistance in the on state, the disturbance from an input through the $\mathrm{CON}$ will be reduced further. If the floating capacitance are metal metal capacitors the CON resistance is equivalent to a bridge resistance between the metal layers. Bridge faults can be divided into two groups, hard or soft, depending on the resistance. Only hard bridge faults may cause problems to a redundant majority-3 gate. If the frequency of the system is very high the extra load will reduce the effect of a CON fault. The response of the majority 3 gate is shown in Figure 4. A ternary 0 implies an error due to a fault.

\subsubsection{Response to faults}

\begin{tabular}{|c|c|c|}
\hline Fault & Output & Response \\
\hline \hline TG short & $\approx V_{D D} / 2$ & 0 \\
\hline TG nMOS open & $\begin{array}{c}\text { reduced }+1 \\
\text { increased }-1 \\
\text { increased recharge delay }\end{array}$ & \pm 1 \\
\hline TG pMOS open & $\begin{array}{c}\text { reduced }-1 \\
\text { increased }+1 \\
\text { increased recharge delay }\end{array}$ & \pm 1 \\
\hline TG open & reduced \pm 1 & $(0)$ \\
\hline nMOS SOFF & $\approx V_{D D}$ & 0 \\
\hline pMOS SOFF & $\approx 0$ (gnd) & 0 \\
\hline nMOS SON & $\approx V_{D D}$ & 0 \\
\hline pMOS SON & $\approx 0$ (gnd) & 0 \\
\hline \hline
\end{tabular}

Table 1. Internal transistor faults in the majority-3 $S F G$ gate and output response. Note that none of the faults will generate an incorrect output transition. No combination of faults will generate a false output transition.

The effect of internal SFG gate faults are given in Table 1. None of the internal faults, or combination of faults, will produce a false output transition. A false output transition can only be observed if the gate receive a false input transition.

The response and output error for different input faults are shown in Table 2. Single and triple SA failures provide

\begin{tabular}{|c|c|c|}
\hline Input fault & Response & Output error \\
\hline \hline Single SA & $\approx V_{D D} / 2$ & 0 (worst case) \\
\hline Double SA & Dep. on a single input & logic \\
\hline Triple SA & $\approx V_{D D} / 2$ & 0 \\
\hline Single CON & $\begin{array}{c}\text { transition dependent } \\
\text { on timing details }\end{array}$ & $(0 /$ none $)$ \\
\hline Double CON & wired or of two inputs & $(0 /$ none $)$ \\
\hline Triple CON & wired or & $(0 /$ none $)$ \\
\hline RBR & wired inputs & $(0 /$ none $)$ \\
\hline \hline
\end{tabular}

Table 2. Input faults and response. An output error equal to 0 implies a ternary 0 . A ternary 0 implies an inactive and not a faulty transition. COFF faults are equivalent to SA faults.

a ternary 0 output which is not as critical as a false transition. For double SA faults the response is determined by the single input which is not SA. In this case the gate operates logically as a SFG single input inverter. If $X$ and $Y$ is SA0 or SA1, which are equivalent to $[X, Y]=[0,0]$, the output will be Output $=-1 \cdot Z$. If for example the correct input should have been $[X, Y, Z]=[+1,+1,-1]$ the gate input will be $[X, Y, Z]=[0,0,-1]$ due to a double SA fault. The response will be Output $=+1$ in contrast to the correct output Output $=-1$.

COFF errors are equivalent to SA errors. CON faults are more critical and timing dependent. The recharge condition is not influenced by $\mathrm{CON}$ faults because all nodes are driven to $V_{D D} / 2$. For a single $\mathrm{CON}$ fault there will be a resistive connection between one of the inputs and the semi-floatinggate. In the case were the two other inputs are not equal the SFG node is directly dominated by the input connected through the resistor. The SFG node will be driven towards the single input through the resistive connection. The delay of the input will be increased due to the resistor and additional capacitances associated with the SFG node including the other input capacitors. If the SFG is unable to response to the change in input the output will be a ternary 0 , otherwise the output will process the correct transition. If however the faultless inputs are equal the only severe problem arises when the resistance between a single input and the SFG node is very small and the operating frequency is very low. In this case the single input may override the other inputs and create a false transition. If a double CON fault is evident ad the resistance of the two resistive connections are not in the same order of magnitude a false output transition may occur in the presence of two nonequal false inputs. If the resistances are approximately equal the two inputs will drive the SFG in opposite directions and the effect is no change of the SFG node due to the CON faults. If all in- 


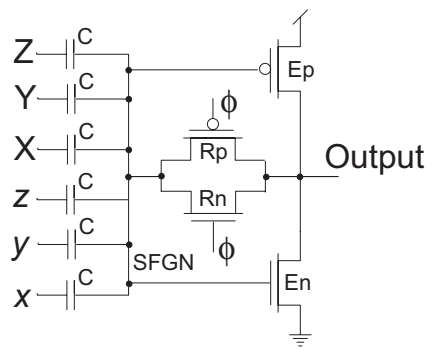

Figure 5. Ternary redundant majority-3 gate.

puts capacitances are $\mathrm{CON}$ and the resistances are equal all inputs will act as wired or connected and a correct output transition will occur.

An input bridge fault (RBR) will effectively reduce the swing of one or two inputs if they experience opposite transitions. An input bridge will not lead to a false transition. Given a suitable operating frequency the MAJ-3 SFG gate is not likely to produce a false transition for any single fault. Only double input SA faults are critical.

\section{Redundant ternary SFG majority-3 gate}

The redundant majority-3 gate is shown in Figure 5. In general, the noise margin will be reduced when increasing the number of inputs. In this case we use a double set of inputs which will not reduce the gain or noise margin. On the other hand, the effect of a CON fault will be further reduced due to a significant increase in delay for the input signal connected to a CON capacitor. The response to input 011 for a simple majority-3 gate (3 inputs) and a redundant majority-3 gate (6 inputs) is shown in Figure 6. The minimum supply voltage for the simple gate is $190 \mathrm{mV}$ yielding a output signal more than $75 \%$ of the input transition and a gain equal to 1.2 for a input transition equal to $V_{D D} / 4=+1 / 2$. The response for a redundant gate assuming that there is a COFF fault or a static input signal is shown in Figure 6. The minimum supply voltage is $250 \mathrm{mV}$. In this case the SFG node voltage change due to a single input signal is $\Delta V_{S F G}=0.15 \cdot V_{D D} / 2=37.5 \mathrm{mV}$. Note that the SFG is balanced in the equilibrium state, given that $I_{E_{p}}=I_{E_{p}}$, when the input signal arises. If the supply voltage is $250 \mathrm{mV}$ the circuit operates in weak inversion and relative transconductance and gain is quite high. If the supply voltage is $1.5 \mathrm{~V}$ the voltage change for the SFGN is $225 \mathrm{mV}$.

The probable effect of one input fault for wired redundancy, MAJ-3 SFG and redundant MAJ-3 SFG gates are shown $i$ Table 3 . A single fault will not influence the operation of the gate. If two input faults are present the worst case scenario is a ternary 0 output which will not affect the following gate.

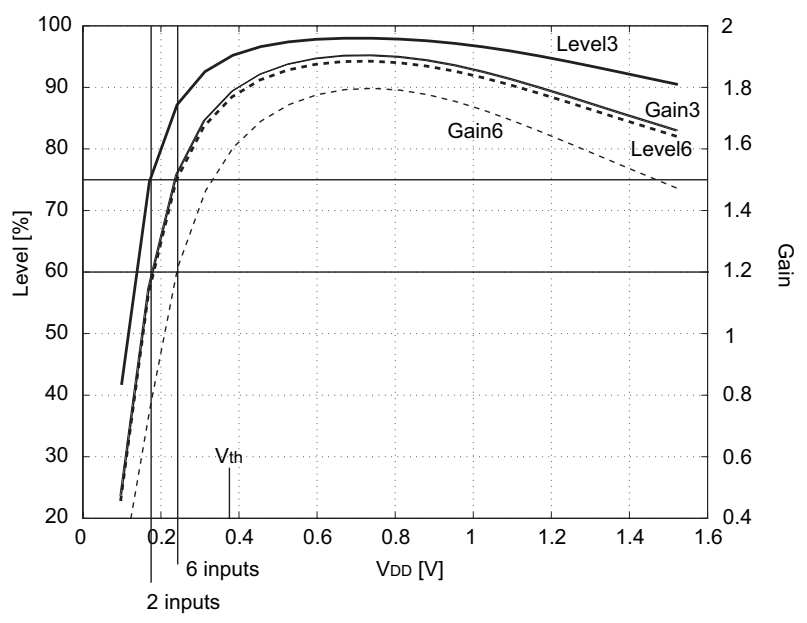

Figure 6. Ternary majority-3 gate response to input $X Y Z$ equal $-1+1+1$. The dashed lines correspond to input $-1+1+1 / 2$ which is modelled as a dc input for $z$ due to an error associated with the gate producing $z$. The minimum supply voltage required for simple majority-3 SFG gate is $190 \mathrm{mV}$ assuming floating input capacitors equal to three times the parasitic capacitance seen bay the semi floatinggate node. By using redundant input signals the minimum supply voltage becomes $250 \mathrm{mV}$.

Simulated (spice and the $0.35 \mu$ AMS CMOS process) response of a redundant MAJ-3 SFG gate to different input faults is shown in Figure 7. The clock frequency is $200 M H z . \mathrm{X}$ (and $x$ ) and Y (and $y$ ) have transitions in opposite directions and the response of the gate is dependent on $\mathrm{Z}$ and $z$. The response to a stuck at fault on the input $z$ is shown as $V_{s a}$ and $\mathrm{Z}$ equal to $\mathrm{Y}$. $V_{s c h 2}$ resembles a response to a hard bridge fault or short circuit of the input capacitor associated with the input $z$. The resistance i parallel to the short circuited capacitor is $100 k \Omega$. $V_{s c s 2}$ resembles a response to a soft bridge fault or short circuit of the input capacitor associated with the input $z$. The resistance i parallel to the short circuited capacitor is $1 M \Omega$. The correct respone of the MAJ-3 gate in this case is to invert the signal $\mathrm{Z}$ (and $z$ ). As can be seen in Figure 7 the redundant MAJ3 gate respond correctly to the input signals at $200 \mathrm{M} \mathrm{Hz}$. For the input SA fault the timing response is affected. Note that the hard bridge fault $(\mathrm{CON})$ has less impact than a soft bridge fault (CON).

Simulated (spice and $0.35 \mu \mathrm{CMOS}$ ) response of a redundant MAJ-3 SFG gate to different input faults is shown in Figure 8. $\mathrm{X}$ (and $x$ ) and $\mathrm{Y}$ (and $y$ ) have transitions in the same direction and the response of the gate is independent on $\mathrm{Z}$ and $z$. $V_{\text {sch }}$ resembles a response to a hard bridge fault or short circuit of the input capacitor associated with the input $z$. $V_{s c s}$ resembles a response to a soft bridge fault or 

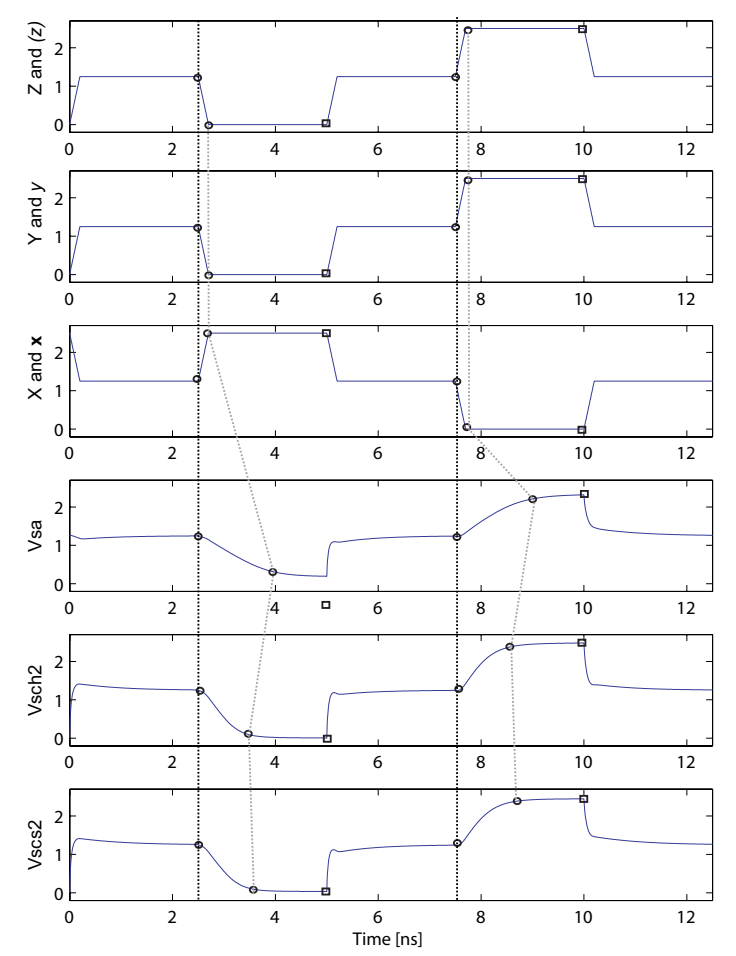

Figure 7. Simulated (spice and the $0.35 \mu$ AMS CMOS process) response of a redundant MAJ-3 SFG gate to different input faults.
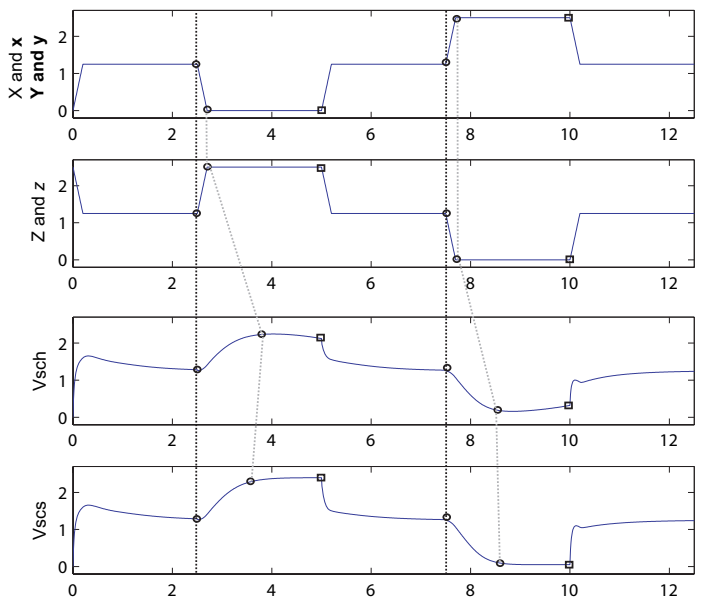

Figure 8. Simulated (spice and $0.35 \mu$ CMOS) response of a redundant MAJ-3 SFG gate to different input faults.

\begin{tabular}{|c|c|c|c|}
\hline Inp. fault & Wired err. & MAJ-3 error & Red. MAJ-3 err. \\
\hline \hline SA & logic & 0 & none \\
\hline RBR & logic & (logic) & none \\
\hline IOFF & none & 0 & none \\
\hline COFF & & logic & none \\
\hline CON & & logic & none \\
\hline \hline
\end{tabular}

Table 3. The probable effect of one input fault for wired redundancy, MAJ-3 SFG and redundant MAJ-3 SFG gates.

short circuit of the input capacitor associated with the input $z$. Given the inputs $\mathrm{X}=x=\mathrm{Y}=y$, the response of the redundant MAJ-3 gate should be unaffected by the thrird input $\mathrm{Z}$ and $z$. Given a clock frequency of $200 \mathrm{MHz}$ the circuit operates correctly. In this case the circuit is less affected by a soft CON fault than by a hard CON fault.

\section{Conclusion}

In this paper we presented fault tolerant CMOS logic using redundancy and ternary signals. Signals are processed through capacitors in such a way that the logic operation of a gate is independent of the DC voltage applied on the inputs. By combining signals through capacitors stuck on/stuck off and stuck at faults are not destructive when redundancy is applied.

\section{References}

[1] T. Shibata and T. Ohmi. "A Functional MOS Transistor Featuring Gate-Level Weighted Sum and Threshold Operations", In IEEE Transactions on Electron Devices, vol 39, 1992.

[2] Y. Berg, S. Aunet, Ø. Næss and O. Mirmotahari. "Basic Multiple-Valued Functions Using Recharge CMOS Logic", Proceedings, 34th Int. Symposium on Multiple-Valued Logic, 2004 .

[3] C. Constantinescu. "Trends and challanges in VLSI circuit reliability", IEEE Micro, Volume: 23, Issue: 4, July-Aug. 2003.

[4] B.W. Johnson. "Design and Analysis of Fault-Tolerant Digital Systems", Addison-Wesley Publishing Compant 1989, ISBN 0-201-07570-9.

[5] K. Kotani, T. Shibata, M. Imai and T. Ohmi. "ClockedNeuron-MOS Logic Circuits Employing Auto-ThresholdAdjustment", In IEEE International Solid-State Circuits Conference (ISSCC), pp. 320-321,388, 1995.

[6] R. Lashevsky, K. Takaara and M. Souma "Neuron MOSFET as a Way to Design a Threshold Gates with the Threshold and Input Weights Alterable in Real Time", IEEE TT13.11-1.4, 1998, pp. 263-266. 